\title{
THE LICK AGN MONITORING PROJECT: ALTERNATE ROUTES TO A BROAD-LINE REGION RADIUS
}

\author{
Jenny E. Greene ${ }^{1,8}$, Carol E. Hood ${ }^{2}$, Aaron J. Barth ${ }^{2}$, Vardha N. Bennert ${ }^{3}$, Misty C. Bentz ${ }^{2,9}$, Alexei V. Filippenko ${ }^{4}$, \\ Elinor Gates $^{5}$, Matthew A. Malkan ${ }^{6}$, Tommaso Treu ${ }^{3,10}$ Jonelle L. Walsh $^{2}$, and Jong-HaK Woo ${ }^{7}$ \\ ${ }^{1}$ Department of Astrophysical Sciences, Princeton University, Princeton, NJ 08544, USA \\ ${ }^{2}$ Department of Physics and Astronomy, 4129 Frederick Reines Hall, University of California, Irvine, CA 92697-4575, USA \\ ${ }^{3}$ Department of Physics, University of California, Santa Barbara, CA 93106, USA \\ ${ }^{4}$ Department of Astronomy, University of California, Berkeley, CA 94720-3411, USA \\ ${ }^{5}$ Lick Observatory, P.O. Box 85, Mount Hamilton, CA 95140, USA \\ ${ }^{6}$ Department of Physics and Astronomy, University of California, Los Angeles, CA 90024, USA \\ ${ }^{7}$ Astronomy Program, Department of Physics and Astronomy, Seoul National University, Seoul, Korea, 151-742 \\ Received 2010 July 7; accepted 2010 September 1; published 2010 October 11
}

\begin{abstract}
It is now possible to estimate black hole $(\mathrm{BH})$ masses across cosmic time, using broad emission lines in active galaxies. This technique informs our views of how galaxies and their central BHs coevolve. Unfortunately, there are many outstanding uncertainties associated with these "virial" mass estimates. One of these comes from using the accretion luminosity to infer a size for the broad-line region (BLR). Incorporating the new sample of low-luminosity active galaxies from our recent monitoring campaign at Lick Observatory, we recalibrate the radius-luminosity relation with tracers of the accretion luminosity other than the optical continuum. We find that the radius of the BLR scales as the square root of the $\mathrm{X}$-ray and $\mathrm{H} \beta$ luminosities, in agreement with recent optical studies. On the other hand, the scaling appears to be marginally steeper with narrow-line luminosities. This is consistent with a previously observed decrease in the ratio of narrow-line to X-ray luminosity with increasing total luminosity. The radius of the BLR correlates most tightly with $\mathrm{H} \beta$ luminosity, while the X-ray and narrow-line relations both have comparable scatter of a factor of 2 . These correlations provide useful alternative virial $\mathrm{BH}$ masses in objects with no detectable optical/UV continuum emission, such as high-redshift galaxies with broad emission lines, radio-loud objects, or local active galaxies with galaxy-dominated continua.
\end{abstract}

Key words: galaxies: active - galaxies: nuclei - galaxies: Seyfert

\section{THE RADIUS-LUMINOSITY RELATION}

Over the past decade, interest in measuring supermassive black hole (BH) masses has intensified, as evidence mounts that BHs play a central role in galaxy evolution (e.g., Silk \& Rees 1998; Hopkins et al. 2006). Locally, BH masses are measured using stars, gas disks, or megamaser disks as dynamical tracers (e.g., Gültekin et al. 2009). None of these techniques can currently reach beyond a few tens of Mpc. Thus, we resort to indirect mass estimates in actively accreting $\mathrm{BHs}$ to probe $\mathrm{BH}$ and galaxy coevolution at cosmological distances. Studies of the BH mass and accretion-rate distributions both locally (Greene \& Ho 2007; Schulze \& Wisotzki 2010) and at higher redshifts (Woo et al. 2006; Kollmeier et al. 2006; Shen et al. 2008b; Woo et al. 2008; Vestergaard \& Osmer 2009; Kelly et al. 2009), as well as studies of possible evolution in $\mathrm{BH}$-bulge scaling relations (e.g., Shields et al. 2003; Treu et al. 2004; Walter et al. 2004; Peng et al. 2006a, 2006b; Treu et al. 2007; Salviander et al. 2007; Alexander et al. 2008; Jahnke et al. 2009; Greene et al. 2010; Bennert et al. 2010), all rely on BH masses derived from active galactic nuclei (AGNs).

$\mathrm{BH}$ masses derived from AGNs use the broad-line region (BLR) gas as the dynamical tracer, based on the assumption that the gas is primarily accelerated by the gravity of the $\mathrm{BH}$. The gas velocity dispersion is derived from the broad-line width, but the $\mathrm{BH}$ mass estimate also requires the radius of the emitting region. The best estimate for its size comes from "reverberation" or echo mapping (Blandford \& McKee 1982). Detailed spectroscopic

\footnotetext{
8 Princeton-Carnegie Fellow.

9 Hubble Fellow.

${ }^{10}$ Sloan Fellow; Packard Fellow.
}

monitoring allows an estimate of the light-travel time through the BLR by measuring the delay between variations in the continuum and line emission (see the recent compilation by Peterson et al. 2004). This technique has a long history (e.g., Antonucci \& Cohen 1983; Peterson et al. 1983; Ulrich et al. 1984; Gaskell \& Sparke 1986), and thus far has yielded reliable sizes for a few dozen sources (see Peterson et al. 2004).

Five reverberation-mapped sources show a $1 / \sqrt{R}$ decline in velocity width ranging from $\mathrm{C}$ IV $\lambda 1549$ to $\mathrm{H} \beta$, as expected for a virialized BLR in a $1 / R$ potential (Kollatschny 2003; Peterson et al. 2004). Data from our Lick AGN Monitoring Project (LAMP), the subject of this paper, are consistent with the same assumption; when multiple Balmer lines are considered independently (e.g., $\mathrm{H} \alpha, \mathrm{H} \beta$, and $\mathrm{H} \gamma$ ), all yield consistent estimates for the so-called virial product, $v^{2} R / G$ (Bentz et al. 2010). On the other hand, other models, such as disk winds, would predict similar radial dependence (e.g., Murray \& Chiang 1995). The importance of radiation pressure in supporting the BLR is currently a matter of debate as well (e.g., Marconi et al. 2008, 2009; Netzer 2009; Netzer \& Marziani 2010). Despite these major uncertainties, the reverberation-derived $\mathrm{BH}$ masses correlate remarkably well with the luminosities and stellar velocity dispersions of their host bulges (Bentz et al. 2009b; Woo et al. 2010). In addition, the very few existing direct dynamical measures of BH masses have so far turned out to be consistent with the reverberation-mapping virial estimates (Davies et al. 2006; Onken et al. 2007; Hicks \& Malkan 2008).

Since reverberation radii are usually not available, a secondary estimate of BLR size is often obtained from the empirical correlation (the "radius-luminosity" relation) between AGN luminosity and BLR size, $R_{\mathrm{BLR}} \propto L^{\beta}$ (Kaspi et al. 2000, 2005; Bentz et al. 2006, 2009a). With just a measurement of the 
AGN luminosity, typically $L_{5100 \AA}$, and a broad-line width, typically $\mathrm{FWHM}_{\mathrm{H} \beta}$, one can roughly estimate a $\mathrm{BH}$ mass as $M_{\mathrm{BH}}=$ $f v^{2} L^{\beta} / G$. Here $f$ is a scaling parameter that includes unknown information about the geometry and kinematics of the BLR.

These so-called single-epoch virial $\mathrm{BH}$ masses are indirect and depend on a number of assumptions. Two empirically determined quantities fundamentally limit the accuracy of the derived $\mathrm{BH}$ masses. One is $f$, which is currently determined for ensembles of active galaxies through comparison between AGN-based masses and other estimates of $M_{\mathrm{BH}}$ such as the $M_{\mathrm{BH}}-\sigma_{*}$ relation (e.g., Gebhardt et al. 2000; Ferrarese et al. 2001; Nelson et al. 2004; Onken et al. 2004; Greene \& Ho 2006; Shen et al. 2008a; Woo et al. 2010). While there are good reasons to suspect that $f$ may depend on physical properties of the $\mathrm{BH}$ such as the accretion rate (e.g., Collin et al. 2006), reverberation-mapping campaigns have not yet succeeded in measuring $f$ directly for individual objects. We are getting closer, however, since two-dimensional reverberation mapping is growing more common and the velocity-resolved emissionline response strongly constrains $f$ in individual sources (e.g., Kollatschny 2003; Bentz et al. 2008; Denney et al. 2009).

The other empirically determined parameter is the slope of the radius-luminosity relation, $\beta$, which is the subject of this paper. We are motivated to revisit this question thanks to our recent reverberation-mapping campaign, which has doubled the number of reverberation-mapped AGNs with $R_{\mathrm{BLR}} \lesssim 10$ lt-day. We do not consider the optical AGN continuum luminosity because the Hubble Space Telescope (HST) is required to spatially disentangle the AGN and galaxy continuum for these low-luminosity sources. The requisite HST imaging is underway (GO-11662, PI: Bentz), and we will present the optical radius-luminosity relation in a future paper. Here, we focus on other direct and indirect indicators of the AGN luminosity, including the X-ray luminosity and broad and narrow emission-line luminosities.

There are practical reasons to consider other routes to determining BLR radii. For example, alternate relations are useful whenever the optical/UV continuum from the AGN is not measurable. This could occur when the AGNs are radio loud, so that the optical/UV continuum is contaminated by synchrotron radiation, or when the galaxy rather than the AGN dominates the optical continuum (e.g., Wu et al. 2004; Greene \& Ho 2005; Arshakian et al. 2010). It has become common practice to use $\mathrm{H} \alpha$ or $\mathrm{H} \beta$ luminosities to calculate $R_{\mathrm{BLR}}$ for high-redshift targets where the continuum is rarely detected (e.g., Alexander et al. 2008; Shapiro et al. 2009). Finally, remarkably, there is indirect evidence that broad-line widths measured from polarized line emission may provide a reasonable single-epoch virial BH mass (e.g., Zhang et al. 2008; Liu et al. 2009, Kuo 2010). In these cases, hard X-rays or narrow emission lines are some of the only available proxies for AGN continuum luminosity.

\subsection{Which Luminosity Best Predicts BLR Size?}

If the spectral energy distributions (SEDs) and the density distribution in the BLR are independent of luminosity, then we expect that the BLR size will scale simply with the square root of the photoionizing luminosity, $R_{\mathrm{BLR}} \propto \sqrt{L}$ (e.g., Netzer 1990). The most recent calibrations of the radius-luminosity relation have all been consistent with this simple relation (Bentz et al. 2006, 2009a).

If the SEDs were really independent of luminosity, the photoionizing luminosity could be estimated from a measure of the AGN continuum at almost any wavelength. However, there are observational indications of luminosity dependence in SEDs.
Indeed, on theoretical grounds we might also expect smaller and hotter accretion disks around lower mass BHs (e.g., Shields 1978; Zheng \& Malkan 1993), which is now testable with observations of gravitationally lensed quasars (e.g., Morgan et al. 2010, Blackburne et al. 2010). While the equivalent width of $\mathrm{H} \beta$ is constant in high-luminosity active galaxies (e.g., Searle \& Sargent 1968), both Croom et al. (2002) and Greene \& Ho (2005) see evidence for a weak inverse-Baldwin effect in $\mathrm{H} \beta$ at low luminosity. Furthermore, the increase in $\alpha_{\text {ox }}$ with UV luminosity (e.g., Avni \& Tananbaum 1982; Steffen et al. 2006; Desroches et al. 2009) suggests luminosity-dependent changes in the SED. Finally, the relative strengths of the "big blue bump" and the $\mathrm{X}$-rays depend on the Eddington ratio, with the latter dominating at lower $L_{\text {bol }} / L_{\text {Edd }}$ (Malkan \& Sargent 1982; Vasudevan \& Fabian 2007; Vasudevan et al. 2009). For a review of the situation at yet lower $L_{\mathrm{bol}} / L_{\mathrm{Edd}}$, see Ho (2008). Therefore, in this paper we will consider several observables which may correlate with, and thus be used to estimate, the ionizing luminosity. Specifically, we consider the following proxies for the AGN luminosity: hard X-ray luminosity $\left(L_{2-10 \mathrm{keV}}\right), \mathrm{H} \beta$ luminosity $\left(L_{\mathrm{H} \beta}\right)$, narrow [O III] $\lambda 5007 \AA$ luminosity $\left.\left(L_{[\mathrm{O}} \mathrm{III}\right]\right)$, and narrow [O IV] $\lambda 25.8 \mu \mathrm{m}$ luminosity $\left(L_{[\mathrm{O} \text { IV }]}\right)$.

Throughout, we assume the following cosmological parameters to calculate distances: $H_{0}=100 \mathrm{~h}=70 \mathrm{~km} \mathrm{~s}^{-1} \mathrm{Mpc}^{-1}$, $\Omega_{\mathrm{m}}=0.30$, and $\Omega_{\Lambda}=0.70$.

\section{THE LICK AGN MONITORING PROJECT}

The new measurements that motivate this work result from LAMP, a dedicated monitoring campaign of 13 AGNs (including the well-studied Seyfert galaxy NGC 5548). We specifically focused on nearby (redshift $z<0.05$ ) Seyfert galaxies with low luminosities $\left(\lambda L_{5100 \AA} \lesssim 10^{43} \mathrm{erg} \mathrm{s}^{-1}\right)$ and probable BH masses in the range $10^{6}-3 \times 10^{7} M_{\odot}$, since this luminosity and mass regime had not been explored fully in the past. Spectroscopic monitoring was carried out with the Lick Observatory $3 \mathrm{~m}$ Shane telescope over a nearly contiguous 64 day period (Bentz et al. 2009c), while photometric monitoring was performed over a longer period utilizing four smaller telescopes (Walsh et al. 2009). We successfully measured BLR radii based on $\mathrm{H} \beta$ for nine objects (Bentz et al. 2009c), reported lags in multiple other Balmer transitions (Bentz et al. 2010), and succeeded in measuring velocity-resolved lags in at least three sources (Bentz et al. 2008, 2009c). Finally, we revisited the calibration of reverberation-mapped $\mathrm{BH}$ masses using the $M_{\mathrm{BH}}-\sigma_{*}$ relation (Woo et al. 2010). For the purpose of this paper, we focus on BLR radii based exclusively on $\mathrm{H} \beta$ lag times.

\section{LUMINOSITIES AND BLR RADII}

The BLR light-crossing times used here are presented by Bentz et al. (2009a) and Bentz et al. (2009c) for the previous reverberation-mapped and LAMP AGNs, respectively. We note that improved lag measurements were more recently reported for a subset of galaxies by Denney et al. (2010). We have confirmed that the radius-luminosity relation based on $\mathrm{H} \beta$ does not change with the inclusion of their lag values, but continue to use the old measurements for temporal consistency with the X-ray observations. We follow Bentz et al. (2009a) and Peterson et al. (2004) and remove IC 4329A from the sample due to uncertainties in the measurements. Throughout, we will refer to the sample of active galaxies with reverberation mapping, excluding the LAMP targets, as the "non-LAMP" objects. We describe the origin of the AGN luminosities in this section 
(Table 1). It is useful to remember that "BLR size" here actually refers to the time of peak response of the $\mathrm{H} \beta$-emitting gas relative to the continuum, multiplied by the speed of light. Had the experiment been done with C IV $\lambda 1549$, for instance, the sizes would have been smaller, but the widths larger. When calculating effective $\mathrm{BH}$ masses, it is important to match the species used to measure velocity dispersion with the radius relation calibrated for the same species.

Of all the luminosities we discuss, only the broad $\mathrm{H} \beta$ line luminosity $\left(L_{\mathrm{H} \beta}\right)$ is measured as part of the reverberationmapping campaign, simultaneously with the radius measurement. It should provide a fairly direct and unbiased probe of the photoionizing continuum. Both Bentz et al. (2009c) and Kaspi et al. (2005) tabulate average $L_{\mathrm{H} \beta}$ measured in the same way. Note that we present results based both on the total $\mathrm{H} \beta$ luminosity (narrow and broad combined) and the broad $\mathrm{H} \beta$ luminosity alone. The results are basically identical, since the median luminosity difference is less than 5\%. Although it would be useful to examine $\mathrm{H} \alpha$ as well, uniform measurements do not exist for the non-LAMP sample, and thus we must await future work.

The [O III] luminosities for the LAMP sample itself are measured from the Shane spectra and are presented by Bentz et al. $(2009 c)$. For the non-LAMP targets, we draw from previous reverberation-mapping campaigns for the local galaxies. For the more distant and luminous Palomar-Green quasars (Schmidt \& Green 1983), we combine the equivalent-width measurements of [O III] from Boroson \& Green (1992) with the continuum fluxes of Kellermann et al. (1989) as given by Ho \& Peng (2001) and Greene et al. (2006). Table 1 contains all measurements, including relevant references. Note that the [O III] luminosities have not been corrected for extinction. The formal errors for the [O III] measurements are in the range 2\%-15\%. However, we find a median difference of $\sim 40 \%$ between different literature values (relying predominantly on the compilation of Whittle 1992). The values used here, from previous reverberation-mapping campaigns, are typically smaller than those compiled by Whittle. Thus, in our fitting we adopt an uncertainty of 0.15 dex in the line luminosities, as an estimate of the impact of various systematic effects discussed below.

A large fraction of the non-LAMP sources have [O IV] $\lambda 25.8 \mu \mathrm{m}$ luminosities available in the literature from the Spitzer Space Telescope. We draw predominantly from the measurements of Dasyra et al. (2008), which cover a large fraction of the non-LAMP reverberation-mapped sample. We take the measurements for Mrk 766 and Mrk 335 from the work of Tommasin et al. (2010) and that of NGC 3516 from the work of Gallimore et al. (2010). With the exception of this last, all were taken with the high-resolution grating. A similar comparative exercise as above, this time with the compilations of Tommasin et al. (2010), Gallimore et al. (2010), and Veilleux et al. (2009), yields a lower uncertainty estimate $(\sim 10 \%)$ than for the [O III] lines. Presumably greater agreement is reached because the data sets were in many cases identical, and so we use a value of $40 \%$ as above.

The X-ray luminosities for the non-LAMP sources are taken directly from the compilation of Kaspi et al. (2005). They are derived from a variety of literature sources, but generally are based on Advanced Satellite for Cosmology and Astrophysics (ASCA) data (Tanaka et al. 1994). While many of these targets have more recent XMM-Newton or Chandra observations available in the archive, the ASCA measurements are actually closer in time to the reverberation-mapping campaign. Thus, we adopt the X-ray luminosities presented by Kaspi et al. in all cases.
The X-ray luminosities for the LAMP objects come from a range of sources. In all cases, we adopt the observation closest in time to that of our campaign (Spring 2008). Six objects (Mrk 142, Apr 151, Mrk 766, Mrk 1310, NGC 5548, and NGC 4748) have X-ray luminosities from Swift. All were observed between 2007 and 2009. The Swift data were extracted using the $x$ select task as part of the HEASARC ${ }^{11}$ tool-set. Each source was extracted from a circular region with a radius of 20 pixels $\left(\sim 47^{\prime \prime}\right)$. Background rates were negligible in all cases. Count rates were converted to fluxes assuming a power-law spectrum with $\Gamma=1.8\left(E \propto E^{-\Gamma}\right)$ and no internal absorption. In the few cases with multiple epochs (e.g., Mrk 766 and NGC 5548), we analyze the longest observation where the galaxy center is close to the image center.

The remainder of the LAMP AGNs only have heterogeneous measurements available in the literature. In one case (NGC 6814) we use the XMM-Newton slew survey (Saxton et al. 2008) and in another (Mrk 202) we resort to an ASCA observation from 1999 (Ueda et al. 2005), where aperture photometry yields a count rate that is converted to a flux assuming only Galactic extinction and $\Gamma=1.7$. The luminosity for NGC 6814 , from the slew survey, was derived in a similar fashion, using the same spectral model. The only difference is that the flux is reported for $2-12 \mathrm{keV}$. We use webPIMMS ${ }^{12}$ to calculate the $2-10 \mathrm{keV}$ flux assuming our spectral model.

With many years of comprehensive monitoring, NGC 5548 is a special case and warrants extra attention. There are 14 nonLAMP epochs from Peterson et al. (2002) and Bentz et al. (2007). In Figure 1(b), we show all 15 epochs in gray for reference. Currently, NGC 5548 is in a very low luminosity state. The LAMP measurement differs by a factor of $\sim 4$ from the weighted average of all other epochs (e.g., Bentz et al. 2009c). Unfortunately, we have only two epochs of X-ray data for this source, and, given narrow-line region (NLR) sizes of hundreds of parsecs, the [O III] luminosity is presumed constant over timescales of months. For the purposes of fitting, we adopt the weighted average lag, $18 \pm 0.6$ lt-day, from Bentz et al. (2009a) as the non-LAMP point. The early X-ray data are from $A S C A$ and were taken in 1993, when the lag was measured to be $13_{-1.4}^{+1.6}$ lt-day (Peterson et al. 2002). If we rather adopt the latter value in our fitting of the X-ray radius-luminosity relation (Section 4), it makes no difference to our results. Since the BLR size of NGC 5548 has been observed to change on timescales short compared to changes in the narrow-line flux, it is also interesting to note that there is a scatter of $0.2 \pm 0.1 \mathrm{dex}$ in the logarithm of the ratio of lag to [O III] luminosity across the 15 epochs.

\subsection{Systematics: X-ray Variability, Aperture Effects, and Extinction}

Each of the luminosities we consider comes with its own complications. In the case of the narrow emission lines, they have been photoionized by the average continuum luminosity over the past $\sim 100$ years, during which time $R_{\mathrm{BLR}}$ may vary significantly. On the other hand, the X-ray emission region is more compact than the optical emitting region, and thus varies on shorter timescales than changes in $R_{\mathrm{BLR}}$ occur. With nonsimultaneous observations, we may introduce significant scatter into the $R_{\mathrm{BLR}}-L_{2-10 \mathrm{keV}}$ relation. In addition, X-ray variability timescales depend systematically on $M_{\mathrm{BH}}$ and luminosity (e.g., O’Neill et al. 2005; McHardy et al. 2006; Miniutti et al. 2009).

\footnotetext{
11 http://heasarc.gsfc.nasa.gov/

12 http://heasarc.gsfc.nasa.gov/Tools/w3 pimms.html
} 
Table 1

Observations

\begin{tabular}{|c|c|c|c|c|c|c|c|}
\hline $\begin{array}{c}\text { Galaxy } \\
\text { (1) }\end{array}$ & $\begin{array}{c}R_{\mathrm{BLR}} \\
(2)\end{array}$ & $\begin{array}{c}L_{2-10 \mathrm{keV}} \\
\text { (3) }\end{array}$ & $\begin{array}{c}L_{\mathrm{H} \beta} \\
(4)\end{array}$ & $\begin{array}{l}L_{[\mathrm{O} \text { III] }} \\
\quad(5)\end{array}$ & $\begin{array}{c}L_{\left[\mathrm{O}_{\text {IV }}\right]} \\
(6)\end{array}$ & $\begin{array}{c}\text { Ref. } \\
(7)\end{array}$ & $\begin{array}{c}\text { Instr., Date } \\
\text { (8) }\end{array}$ \\
\hline Mrk142 & $2.9 \pm 1.3$ & 42.1 & $41.64 \pm 0.04$ & 41.2 & 1 & $\ldots$ & S, 2007 Nov 24 \\
\hline SBS1116 & $2.4 \pm 1.3$ & $\ldots$ & $40.67 \pm 0.04$ & 40.4 & 1 & $\ldots$ & $\ldots$ \\
\hline Arp151 & $4.1 \pm 1.2$ & 42.5 & $40.97 \pm 0.07$ & 40.7 & 1 & $\ldots$ & $S, 2009$ Feb 21 \\
\hline Mrk1310 & $3.7 \pm 1.2$ & 42.3 & $40.62 \pm 0.04$ & 41.0 & 1 & $\ldots$ & S, 2007 Aug 7 \\
\hline Mrk202 & $3.1 \pm 1.6$ & 42.4 & $40.48 \pm 0.04$ & 40.4 & 1 & $\ldots$ & A, 1999 Nov 9 \\
\hline Mrk766 & $6.2 \pm 1.3$ & 42.9 & $40.87 \pm 0.02$ & 41.3 & 1 & 41.2 & $S, 2006$ Dec 28 \\
\hline NGC4748 & $5.6 \pm 1.5$ & 41.3 & $41.01 \pm 0.02$ & 41.2 & 1 & $\ldots$ & $S, 2007$ Jan 8 \\
\hline NGC5548 & $4.3 \pm 1.3$ & 43.4 & $41.35 \pm 0.04$ & 41.6 & 1 & $\ldots$ & $S, 2007$ Jun 19 \\
\hline NGC6814 & $6.7 \pm 1.1$ & 42.2 & $40.23 \pm 0.04$ & 40.0 & 1 & $\ldots$ & $X, 2003$ Oct 19 \\
\hline Mrk335 & $15.7 \pm 1.3$ & 43.2 & $42.14 \pm 0.01$ & 41.5 & 2 & 41.0 & \\
\hline PG0026+129 & $111.0 \pm 1.3$ & 44.5 & $42.92 \pm 0.04$ & 42.9 & 3 & 42.2 & \\
\hline PG0052+251 & $89.8 \pm 1.3$ & 44.8 & $43.12 \pm 0.05$ & 42.8 & 3 & $\ldots$ & \\
\hline Fairall9 & $17.4 \pm 1.3$ & 44.0 & $42.65 \pm 0.02$ & 41.9 & 4 & 41.5 & \\
\hline Mrk590 & $25.6 \pm 1.1$ & 43.6 & $41.79 \pm 0.05$ & 41.2 & 2 & 40.7 & \\
\hline $3 \mathrm{C} 120$ & $38.1 \pm 1.6$ & 44.0 & $42.36 \pm 0.04$ & 41.9 & 2 & 42.5 & \\
\hline Ark120 & $39.7 \pm 1.1$ & 43.8 & $42.50 \pm 0.03$ & 41.3 & 2 & $\ldots$ & \\
\hline Mrk79 & $15.2 \pm 1.4$ & 43.5 & $41.88 \pm 0.03$ & 41.5 & 2 & $\ldots$ & \\
\hline PG0804+761 & $146.9 \pm 1.1$ & 44.3 & $43.26 \pm 0.03$ & 42.3 & 3 & 41.7 & \\
\hline PG0844+349 & $32.3 \pm 1.6$ & 43.4 & $42.52 \pm 0.04$ & 41.6 & 3 & 41.2 & \\
\hline Mrk110 & $25.5 \pm 1.2$ & 43.9 & $42.02 \pm 0.02$ & 41.8 & 2 & $\ldots$ & \\
\hline PG0953+414 & $150.1 \pm 1.2$ & 44.6 & $43.43 \pm 0.02$ & 43.0 & 3 & 42.3 & \\
\hline NGC3227 & $7.8 \pm 3.8$ & 41.9 & $40.49 \pm 0.05$ & 40.4 & 5 & 40.3 & \\
\hline NGC3516 & $6.7 \pm 2.2$ & 43.1 & $41.33 \pm 0.05$ & 40.8 & 5 & 41.0 & \\
\hline NGC3783 & $10.2 \pm 1.3$ & 43.0 & $41.49 \pm 0.03$ & 41.2 & 6 & 40.9 & \\
\hline NGC4051 & $5.8 \pm 1.4$ & 41.4 & $39.70 \pm 0.08$ & 39.7 & 7 & 39.6 & \\
\hline NGC4151 & $6.6 \pm 1.2$ & 42.7 & $41.10 \pm 0.09$ & 41.6 & 8 & 40.8 & \\
\hline PG1211+143 & $93.8 \pm 1.5$ & 43.7 & $42.99 \pm 0.05$ & 42.4 & 3 & 41.4 & \\
\hline PG1226+023 & $306.8 \pm 1.3$ & 45.8 & $44.10 \pm 0.03$ & 43.0 & 3 & 42.8 & \\
\hline PG1229+204 & $37.8 \pm 1.7$ & 43.4 & $42.31 \pm 0.05$ & 41.9 & 3 & 41.4 & \\
\hline NGC4593 & $3.7 \pm 1.2$ & 42.8 & $40.89 \pm 0.11$ & 40.6 & 9 & 40.4 & \\
\hline PG1307+085 & $105.6 \pm 1.5$ & 44.4 & $43.14 \pm 0.05$ & 42.8 & 3 & 42.1 & \\
\hline Mrk279 & $16.7 \pm 1.3$ & 43.7 & $41.96 \pm 0.04$ & 41.5 & 10 & 41.4 & \\
\hline PG1411+442 & $124.3 \pm 1.7$ & 43.2 & $42.83 \pm 0.02$ & 42.0 & 3 & 41.6 & \\
\hline NGC5548 & $18.0 \pm 1.0$ & 43.5 & $41.73 \pm 0.05$ & 41.6 & 11 & 40.9 & \\
\hline PG1426+015 & $95.0 \pm 1.5$ & 44.0 & $42.80 \pm 0.04$ & 42.2 & 3 & 41.9 & \\
\hline Mrk817 & $21.8 \pm 1.1$ & $\ldots$ & $41.97 \pm 0.03$ & $\ldots$ & 2 & $\ldots$ & \\
\hline PG1613+658 & $40.1 \pm 1.5$ & 44.4 & $42.98 \pm 0.03$ & 42.4 & 3 & 42.4 & \\
\hline PG1617+175 & $71.5 \pm 1.6$ & $\ldots$ & $42.71 \pm 0.05$ & $\ldots$ & 3 & $\ldots$ & \\
\hline PG1700+518 & $251.8 \pm 1.2$ & $\ldots$ & $43.74 \pm 0.02$ & $\ldots$ & 3 & $\ldots$ & \\
\hline 3С 390.3 & $23.6 \pm 1.3$ & 44.2 & $42.29 \pm 0.03$ & $\ldots$ & 12 & $\ldots$ & \\
\hline Mrk509 & $79.6 \pm 1.1$ & 44.1 & $42.60 \pm 0.00$ & 42.3 & 2 & 41.9 & \\
\hline PG2130+099 & $22.9 \pm 1.2$ & 43.7 & $42.74 \pm 0.04$ & 42.1 & 3 & 42.0 & \\
\hline NGC7469 & $4.5 \pm 1.2$ & 43.2 & $41.76 \pm 0.02$ & 41.7 & 13 & 41.4 & \\
\hline
\end{tabular}

Notes. Column 1: Galaxy name. Note that references for all data sets are included in Section 3. Column 2: BLR radius (light days). Column 3: X-ray luminosity ( $\mathrm{erg} \mathrm{s}^{-1}$ ). Errors are not included, since they are dominated by variability, but we assume $20 \%$ uncertainties on all values (see Section 3.1). Column 4: H $\beta$ luminosity (erg s ${ }^{-1}$ ). Column 5 : [O III] $\lambda 5007 \AA$ luminosity (erg s ${ }^{-1}$ ); assumed errors are $0.15 \mathrm{dex}$, based on differences in literature measurements (Section 3). Column 6: references for the [O III] fluxes: (1) Bentz et al. 2009c; (2) Peterson et al. 1998; (3) Boroson \& Green 1992; (4) Winge et al. 1996; (5) Denney et al. 2010; (6) Stirpe et al. 1994; (7) Peterson et al. 2000; (8) Kaspi et al. 1996; (9) Dietrich et al. 1994; (10) Santos-Lleó et al. 2001; (11) Peterson et al. 1991; (12) Dietrich et al. 1998; (13) Collier et al. 1998. Column 7: [O IV] $\lambda 25.8 \mu \mathrm{m}$ luminosity ( $\mathrm{erg} \mathrm{s}^{-1}$ ); assumed errors are $0.15 \mathrm{dex}$ as for [O III] above. Column 8: instrument for X-ray luminosity: $S=S$ wift, $C=$ Chandra, $X=X M M-N e w t o n, A=A S C A$. Below the line, all X-ray luminosities are taken from Kaspi et al. (2005). For the LAMP objects, we also include the date of observation.

Thus, it is at least conceivable that some systematic bias is introduced into the $R_{\mathrm{BLR}}-L_{2-10 \mathrm{keV}}$ relation. We investigate that possibility here.

We start by considering all multi-epoch data available for reverberation-mapped sources from the Tartarus database. ${ }^{13}$ The benefit of Tartarus is that the fluxes have been derived from

13 http://tartarus.gsfc.nasa.gov/ the ASCA X-ray spectra in a uniform way. Spectral fits to the hard X-rays (2-10 keV) are performed, with the region around $\mathrm{Fe} \mathrm{K} \alpha$ masked and including possible internal absorption (which is small in this spectral region). Ten of the non-LAMP targets have multiple epochs of observations spanning more than one year in the Tartarus database. They include $3 \mathrm{C} 120$, Fairall 9, Mrk 509, NGC 3227, NGC 3516, NGC 3783, NGC 4051, NGC 4151, NGC 4593, NGC 5548, NGC 4269, and PG 

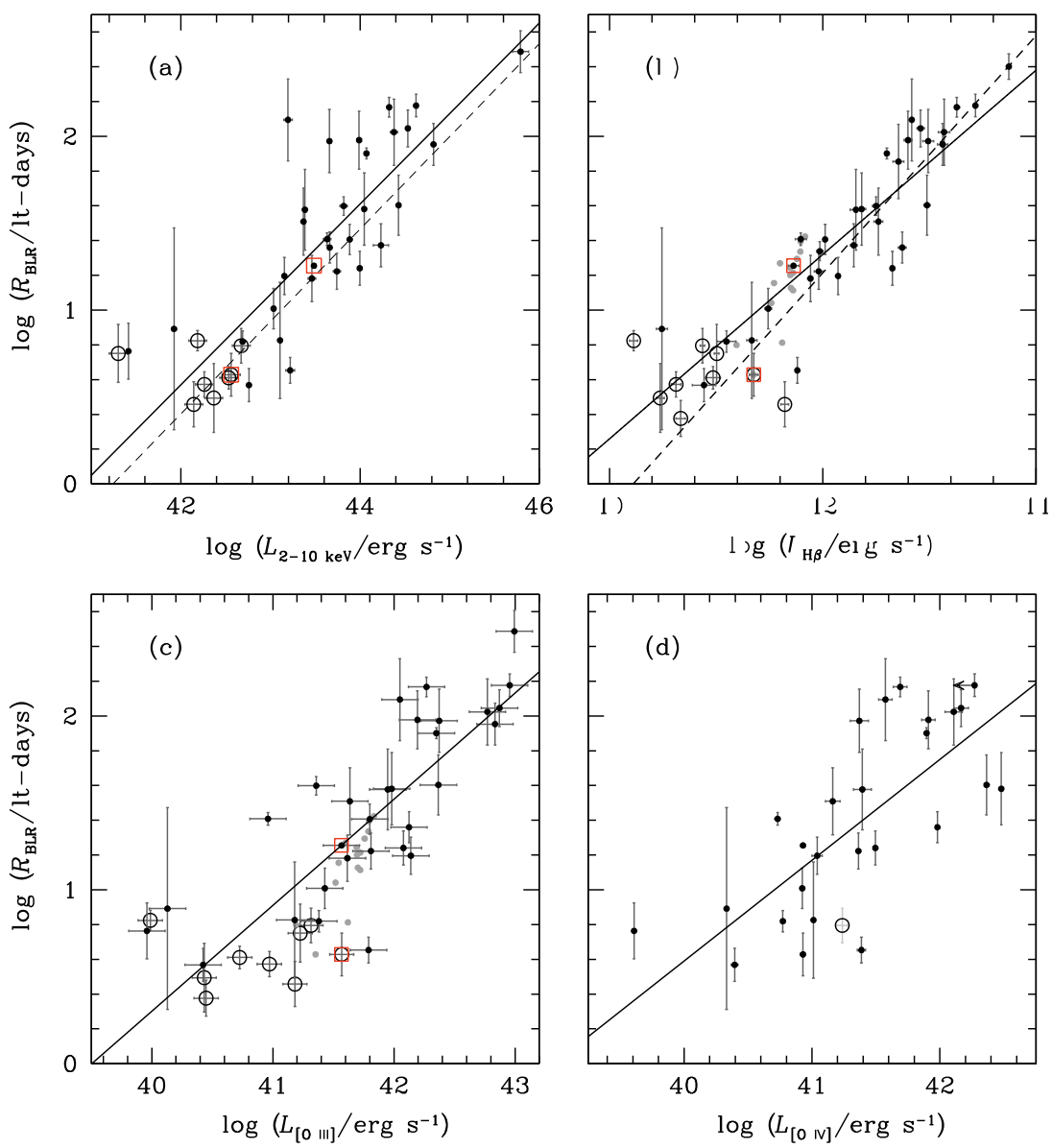

Figure 1. (a) Fit to the $L_{2-10 \mathrm{keV}}-R_{\mathrm{BLR}}$ relation, including both LAMP (open circles) and non-LAMP sources (filled dots). Our maximum-likelihood fit (solid; $\beta=0.52 \pm 0.05$ ) and the fit of Kaspi et al. (2005; dashed) agree in this case. The intrinsic scatter (Table 2) is $\sim 0.3$ dex. NGC 5548 is included twice and indicated with a red box. (b) As in (a), but here using the $\mathrm{H} \beta$ rather than $\mathrm{X}$-ray luminosity. In this case, our maximum-likelihood fit (solid; $\beta=0.53 \pm 0.04$ ) is significantly shallower than that of Kaspi et al., bringing the slope into agreement with that of Bentz et al. (2009a) for the optical continuum. For reference, we show all 15 epochs of monitoring for NGC 5548 in gray. (c) The $R_{\mathrm{BLR}}-L_{[\mathrm{O} \text { II] }}$ relation. As above, LAMP sources are open circles, while the non-LAMP sources are small black circles. The solid line is our best maximum-likelihood fit ( $\beta=0.60 \pm 0.07$ ). (d) As in (c), but here using the [O IV] $25.8 \mu$ m luminosity rather than the [O III] luminosity. The arrow indicates the upper limit on the [O IV] luminosity of PG2130 + 099. Our maximum-likelihood fit $(\beta=0.69 \pm 0.13)$ is shown as a solid line.

$1226+023$. The typical cadence is a few observations per year.

For each object, we calculate a mean and root-mean-square (rms) flux using the Tartarus database. We find variability amplitudes of 5\%-80\% (one standard deviation) over the $1-7 \mathrm{yr}$ timescales probed by these observations. NGC 3516 is the target with the highest variability amplitude $(80 \%)$. The majority of objects do not vary even by a factor of 2 on these timescales. The median amplitude of variability is $\sim 20 \%$. Thus, the level of intrinsic variability in the X-ray luminosity of most Seyferts is usually too small to impact the $R_{\mathrm{BLR}}-L_{2-10 \mathrm{keV}}$ relation. For fitting purposes, we thus adopt $20 \%$ uncertainties in all X-ray fluxes.

We perform a second check using artificial light curves. Our goal is to investigate whether systematic changes in break timescale will lead to a bias in our derived $R_{\mathrm{BLR}}-L_{2-10 \mathrm{keV}}$ relation. We use the prescriptions of Timmer \& Koenig (1995) to generate mock light curves with an input power spectrum of variability. We generate a family of light curves, each of 5 yr duration, and each with a characteristic break in the power spectral density function. The break timescales range from 0.01 to 30 days, which is similar to the range of $0.005-30$ days seen in reverberation-mapped sources (e.g., Uttley \& McHardy $2005)$. For simplicity, all light curves have a power-law slope of $\alpha=-2\left(P \propto f^{\alpha}\right)$ at frequencies above the break frequency and a slope of $\beta=-1\left(P \propto f^{\beta}\right)$ at frequencies below the break. Each artificial light curve is "observed" 2000 times with $10 \mathrm{ks}$ duration. The signal-to-noise ratio is taken to be 100 (but does not impact the results), and the assumed variability amplitude is $20 \%$ to match the Tartarus average above. We then look at the spread in derived X-ray fluxes as a function of break timescale. There is no change in the width of the distribution of mean fluxes for breaks ranging from 0.01 to 30 days. Therefore, we do not expect any systematic errors in our X-ray flux estimates as a function of mass or luminosity based on trends between break timescale and mass or Eddington ratio (McHardy et al. 2006).

It is also worth discussing the primary sources of systematic errors in the fluxes of narrow emission lines. First, aperture corrections can be significant, since NLRs have sizes of hundreds of parsecs and are often spatially resolved (e.g., Whittle 1992; Bennert et al. 2002; Schmitt et al. 2003a, 2003b; Greene et al. 2009). Objects that are closer are more susceptible to aperture effects. The LAMP targets were observed with a slit of width $4^{\prime \prime}$, while the non-LAMP targets generally come from apertures of width $2^{\prime \prime}-20^{\prime \prime}$ (although most are larger than $4^{\prime \prime}$ ), and the Boroson \& Green (1992) observations were taken with a 1".5 slit. Between the compilations of Bennert et al. (2002) and Schmitt et al. (2003a), we find NLR sizes for four of the PG quasars, two LAMP objects (NGC 5548 and Mrk 766), and five other 
non-LAMP objects (Mrk 590, NGC 3516, Mrk 79, NGC 3783, and NGC 4593). The NLR sizes of these galaxies are $0.1^{\prime \prime}-2^{\prime \prime}$, with a median size of 0.3 . The PG quasars all have NLR sizes $<1$.'2. Nominally, based on the objects with measured NLR sizes, we expect minimal loss of light due to aperture effects. Observing conditions will also lead to some slit losses, in the case of the Boroson \& Green (1992) observations, but not at a level that is significant compared to internal extinction (see below). To address the rest of the sample, we estimate NLR sizes using the size-luminosity relation of Schmitt et al. (2003b). We find that the typical expected NLR size (for both LAMP and non-LAMP sources) is $\sim 1$ 1.3. The PG quasars, which were observed with the smallest slit, all have expected sizes in the range $0{ }^{\prime} .5-1^{\prime \prime}$. We are in even less danger with the $L_{\left[\mathrm{O}_{\text {Iv }}\right]}$ measurements, given their larger aperture sizes $\left(5^{\prime \prime}-20^{\prime \prime}\right)$ and compact emission regions (Meléndez et al. 2008).

The next important concern is internal extinction. It has been shown many times, particularly for obscured AGNs, that the [O III] luminosity can be significantly extinguished by dust (e.g., Malkan 1983; Mulchaey et al. 1994; Netzer et al. 2006; Meléndez et al. 2008). On the other hand, $L_{\left[\mathrm{O}_{\text {Iv }}\right]}$ is relatively insensitive to extinction, making it a higher fidelity luminosity indicator (e.g., Meléndez et al. 2008; Diamond-Stanic et al. 2009); if the $L_{[\mathrm{O} \mathrm{m}]}$ measurements are compromised, we still expect to find reasonable results for $L_{[\mathrm{O} \text { iv] }}$. Finally, there is the possibility of contamination from star formation. In principle, this is possible for $\left.L_{[\mathrm{O}} \mathrm{III}\right]$, but note that the $[\mathrm{O} \mathrm{III}] / \mathrm{H} \beta$ intensity ratio is considerably lower in high-metallicity star-forming galaxies than in active galaxies (e.g., Baldwin et al. 1981). As shown by Kauffmann et al. (2003), the expected level of $L_{\text {[О III] }}$ contamination from star formation in local obscured active galaxies is low $(<10 \%)$.

\section{FITS}

Our primary goal is to calibrate the relation between BLR size and various indicators of nonstellar (AGN) luminosity. We fit to the standard relation $\left(R_{\mathrm{BLR}} / 10\right.$ lt-day $)=\alpha+\beta \log L$, where $L$ here is derived from $\mathrm{H} \beta, L_{2-10 \mathrm{kev}}$, and narrow emissionline luminosities. For comparison with the recent literature, we utilize two primary fitting schemes. The first is a $\chi^{2}$ minimization technique similar to that presented by Tremaine et al. (2002). The following $\chi^{2}$ function is minimized:

$$
\chi^{2} \equiv \sum_{i=1}^{N} \frac{\left(R_{i}-\alpha-\beta L_{i}\right)^{2}}{\epsilon_{R i}^{2}+\beta^{2} \epsilon_{L i}^{2}} .
$$

Intrinsic scatter is accounted for by replacing $\epsilon_{R i}$ with $\epsilon=$ $\left(\epsilon_{R i}^{2}+\epsilon_{0}^{2}\right)^{1 / 2}$, where $\epsilon_{0}$ (the intrinsic scatter) is chosen such that $\chi_{r}^{2}=1$.

In addition, we use a maximum-likelihood technique adapted from Gültekin et al. (2009). For simplicity, we assume that both the measurement errors and the intrinsic scatter have Gaussian distributions. For a set of observed points $\left(R_{i}, L_{i}\right)$, we maximize the total likelihood,

$$
\mathcal{L}=\prod_{i} l_{i}\left(R_{i}, L_{i}\right)
$$

In the presence of measurement errors, if the likelihood of measuring a BLR radius $R_{i}$ for a true radius $R$ is $Q_{i}\left(R_{i} \mid R\right) d R_{i}$, and the probability to have a true radius $R$ given $L_{i}$ is $P$, then for
Table 2

Fits

\begin{tabular}{lcccc}
\hline \hline \multicolumn{1}{c}{$L$} & $\alpha$ & $\beta$ & $\epsilon_{0}$ & Fit \\
\multicolumn{1}{c}{$(1)$} & $(2)$ & $(3)$ & $(4)$ & $(5)$ \\
\hline$L_{2-10 \mathrm{keV}} / 10^{43}$ & $0.09 \pm 0.05$ & $0.52 \pm 0.05$ & 0.26 & $\mathrm{C}$ \\
$L_{2-10 \mathrm{keV}} / 10^{43}$ & $0.09 \pm 0.06$ & $0.52 \pm 0.05$ & $0.25 \pm 0.05$ & $\mathrm{ML}$ \\
$L_{\mathrm{H} \beta} / 10^{43}$ & $0.85 \pm 0.05$ & $0.53 \pm 0.04$ & 0.22 & $\mathrm{C}$ \\
$L_{\mathrm{H} \beta} / 10^{43}$ & $0.85 \pm 0.06$ & $0.53 \pm 0.04$ & $0.22 \pm 0.04$ & $\mathrm{ML}$ \\
$L_{\mathrm{H} \beta_{\mathrm{b}}} / 10^{43}$ & $0.86 \pm 0.06$ & $0.53 \pm 0.04$ & 0.22 & $\mathrm{C}$ \\
$L_{\mathrm{H} \beta_{\mathrm{b}}} / 10^{43}$ & $0.86 \pm 0.06$ & $0.53 \pm 0.04$ & $0.22 \pm 0.04$ & $\mathrm{ML}$ \\
$L_{\left[\mathrm{O}_{\text {III }}\right]} / 10^{42}$ & $0.53 \pm 0.06$ & $0.62 \pm 0.07$ & 0.29 & $\mathrm{C}$ \\
$L_{\left[\mathrm{O}_{\text {III }}\right]} / 10^{42}$ & $0.52 \pm 0.06$ & $0.61 \pm 0.07$ & $0.30 \pm 0.05$ & $\mathrm{ML}$ \\
$L_{\left[\mathrm{O}_{\text {IV }}\right]} / 10^{42}$ & $0.76 \pm 0.10$ & $0.58 \pm 0.11$ & 0.35 & $\mathrm{C}$ \\
$L_{\left[\mathrm{O}_{\text {IV }}\right]} / 10^{42}$ & $0.75 \pm 0.10$ & $0.58 \pm 0.11$ & $0.35 \pm 0.07$ & $\mathrm{ML}$ \\
\hline
\end{tabular}

Notes. Fits to $\log \left(R_{\mathrm{BLR}} / 10 \mathrm{pc}\right)=\alpha+\beta \log L$, for each luminosity. Column 1 : luminosity measure $\left(\mathrm{erg} \mathrm{s}^{-1}\right) . L_{\mathrm{H} \beta_{\mathrm{b}}} / 10^{43}$ has the narrow $\mathrm{H} \beta$ emission removed. Column 2: $\alpha$. Column 3: $\beta$. Column 4: intrinsic scatter. Maximum-likelihood fits are those with error bars on the intrinsic scatter. Column 5: fit type, either $\mathrm{C}=\chi^{2}$ fit or $\mathrm{ML}=$ maximum-likelihood.

a given observation the likelihood is

$$
l_{i}=\int Q_{i}\left(R_{i} \mid R\right) P\left(R \mid L_{i}\right) d L .
$$

We assume that both $Q$ and $P$ have a log-normal form. Uncertainties in the independent variable (luminosity) are derived from Monte Carlo simulations and are always small. Fits using both methods are given in Table 2, with the first line showing the $\chi^{2}$ method. In all cases the results of the two fitting methods are indistinguishable.

It is interesting to note that the $\mathrm{X}$-ray and $\mathrm{H} \beta$ relations are now consistent with a slope of $R_{\mathrm{BLR}} \propto \sqrt{L}$. In contrast, Kaspi et al. (2005) report a slope of 0.7 for the X-ray relation $\left(R_{\mathrm{BLR}} \propto L_{2-10 \mathrm{keV}}^{0.7}\right)$. We should note, however, that when they fit an average lag for each object and used only $\mathrm{H} \beta$ lags (the most directly comparable case to what we have done here), they find a slope of $0.5\left(R_{\mathrm{BLR}} \propto L_{2-10 \mathrm{keV}}^{0.53}\right)$. Their reported $R_{\mathrm{BLR}}-L_{\mathrm{H} \beta}$ slope is steeper, $R_{\mathrm{BLR}} \propto L_{\mathrm{H} \beta}^{0.69}$. With our improved data, we find that both relations are consistent with a slope of 0.5 . Thus, the simplest assumption, that AGN SEDs and BLR densities are independent of luminosity, appears to apply, at least for the present sample and to the level of precision that can be tested by our data. One goal of ongoing reverberation-mapping campaigns should be to investigate whether there are physical regimes (e.g., in BH mass or luminosity) for which this assumption does not hold (e.g., Greene \& Ho 2009).

There is tantalizing evidence, in contrast, that the narrow-line relations may have a steeper slope, although with low significance. Here, we explore possible interpretations of this result, should it turn out to be significant. Above we discussed various sources of contamination of the NLR luminosity, including redshift-dependent aperture correction, extinction, and star formation. Aperture effects go in the wrong direction to explain the steeper slope, while extinction seems implausible because it would have to impact the $L_{[\mathrm{O} \text { Iv }]}$ measurements as strongly as the $L_{[\mathrm{O} \mathrm{III}]}$ measurements, contrary to normal reddening laws. Star formation could artificially boost the NLR luminosities at the low end. However, we do not believe the $L_{[\mathrm{OIII}}$ contamination could be more than $\sim 10 \%$ on average, while the values need to be boosted by factors of 2-3 to impact the slope on a logarithmic scale. Therefore, the steeper slope, if real, is more likely explained by physical effects rather than measurement 
errors. It would most naturally arise from the measured luminosity dependence in the relation between NLR and bolometric luminosity. We are not the first to report this trend. For instance, Netzer et al. (2006) find that $L_{[\mathrm{O} \text { III }]} \propto L_{2-10 \mathrm{keV}}^{0.70 \pm 0.06}$, while Meléndez et al. (2008) find $L_{[\mathrm{O} \text { IV }]} \propto L_{2-10 \mathrm{keV}}^{0.7 \pm 0.1}$ and $L_{[\mathrm{OIII}} \propto L_{[\mathrm{OIV}]}^{0.9 \pm 0.1}$. If $R_{\mathrm{BLR}} \propto L_{2-10 \mathrm{keV}} 0.5$, then based on Netzer et al. we would expect $R_{\mathrm{BLR}} \propto L_{[\mathrm{O} \text { III }]} 0.7 \pm 0.1$, which is consistent with our finding. The slope we measure in the $R_{\mathrm{BLR}}-L_{[\mathrm{O} \text { Iv] }}$ relation is also consistent with the results of Meléndez et al. (2008). Thus, the possibility of a steeper slope is plausible. For some reason, quasars are less efficient at powering an NLR than are the less luminous Seyfert nuclei.

There is now compelling evidence that bolometric corrections depend on the Eddington ratio $L_{\text {bol }} / L_{\text {Edd }}$, where the Eddington luminosity for $1 M_{\odot}$ is taken to be $1.25 \times 10^{38} \mathrm{erg}$ $\mathrm{s}^{-1}$ (e.g., Vasudevan \& Fabian 2007; Vasudevan et al. 2009). It is worth seeking correlations between radius-luminosity relation residuals and the Eddington ratio. $\mathrm{BH}$ mass measurements are provided by the reverberation-mapping campaigns. In the case of the non-LAMP sources, we take the Eddington ratios from the study of Vasudevan et al. (2009), who have measured simultaneous SEDs ranging from the optical to the X-ray using $X M M-N e w t o n$. We do not yet have full SEDs for the LAMP sample, and so we use a single-band observation and a bolometric correction. We adopt $L_{2-10 \mathrm{keV}}$ and the bolometric correction from Vasudevan et al. (2009). The bolometric correction depends on the Eddington ratio and we assume a value of 30 , as appropriate for sources with $L_{\mathrm{bol}} / L_{\mathrm{Edd}} \approx 10 \%$. The resulting Eddington ratios are in the range 0.001-1, but are strongly peaked at $\sim 0.1$.

We seek correlations between the residuals in $R_{\mathrm{BLR}}$ around the mean $R_{\mathrm{BLR}}-L$ relations and the Eddington ratio. The nonparametric Kendall's $\tau$ is calculated (within $\operatorname{IRAF}^{14}$ ) for all relations. In no case do we find evidence for a correlation between the $R_{\mathrm{BLR}}-L$ residuals and $L_{\mathrm{bol}} / L_{\mathrm{Edd}}$. The probability of no correlation is in the range $P=0.3-0.8$.

Although we do not know its origin, it is interesting to examine the intrinsic scatter for each fit. As expected, the intrinsic scatter is lowest when $L_{\mathrm{H} \beta}$ is used, presumably because of both temporal and spatial coincidence. On the other hand, the relations based on both the X-rays and narrow emission lines have comparable scatter. One might expect higher scatter in the narrow emission-line relation due to the unquantified role of internal extinction and aperture effects. Furthermore, the narrow-line emission cannot respond to changes in accretion luminosity on timescales of a month, while we know that $R_{\mathrm{BLR}}$ does. Note that NGC 5548 has shown $R_{\mathrm{BLR}}$ variability at the factor of four level, and yet the overall relations only have an intrinsic scatter of a factor of 2 . Once the $L_{5100 \AA}$ measurements are in hand, it will be interesting to see whether the intrinsic scatter is minimized using the optical continuum luminosity or, indeed, the bolometric luminosity.

\section{SUMMARY}

We explore radius-luminosity relations based on AGN luminosities other than the optical continuum. The time is right to revisit these relations because of a new sample of low-luminosity, low-mass AGNs with reverberation mapping from the LAMP project (Bentz et al. 2009c). We consider X-ray, broad $\mathrm{H} \beta$, narrow [O III], and narrow [O IV] luminosities. These relations are

\footnotetext{
14 http://iraf.noao.edu/
}

designed for use in estimating BH masses when optical continuum luminosities are not available. Relevant situations include local AGNs with galaxy-dominated spectra, and possibly radioloud objects, various high-redshift active galaxy populations (such as submillimeter galaxies), and heavily obscured AGNs with detected broad polarized emission. Furthermore, any differences in slope or intrinsic scatter between relations based on different luminosities may indicate SED differences in AGNs as a function of luminosity or $\mathrm{BH}$ mass.

We find that the $R_{\mathrm{BLR}}-L_{2-10 \mathrm{keV}}$ and $R_{\mathrm{BLR}}-L_{\mathrm{H} \beta}$ relations are well fit with a slope of $R_{\mathrm{BLR}} \propto \sqrt{L}$. This is the slope expected if AGN SEDs and BLR densities are independent of luminosity. On the other hand, the narrow emission lines show tentative evidence for a steeper relation, $R_{\mathrm{BLR}} \propto L^{0.6}$. Intriguingly, these slopes are consistent with previous results showing that $L_{[\mathrm{O}}{ }_{\mathrm{III}} /$ $L_{\mathrm{X}}$ and $L_{[\mathrm{O} \text { Iv }]} / L_{\mathrm{X}}$ decrease with increasing luminosity (e.g., Netzer et al. 2006; Meléndez et al. 2008). We find no evidence for a correlation between $R_{\mathrm{BLR}}-L$ residuals and Eddington ratio. In fact, the intrinsic scatter in all relations is surprisingly small. On the one hand, the X-rays are variable on short timescales, but, as we show, that does not translate into significant errors in the $R_{\mathrm{BLR}}-L_{2-10 \mathrm{keV}}$ relation. On the other hand, the narrow emission-line luminosities do not respond at all to state changes on timescales of a year. Thus, we find it surprising that even in these cases the intrinsic scatter is only at the factor of two level. Still, this scatter translates directly into uncertainties in the BH masses (e.g., Vestergaard \& Peterson 2006; McGill et al. 2008). As the reverberation-mapped samples increase, it should become possible to search for evidence of secondary parameters that might allow one to decrease the total scatter, thereby increasing the fidelity of our BH mass estimates.

The referee gave many valuable comments that substantially improved this manuscript. We thank the excellent staff and support personnel at Lick Observatory for their enormous help during our observing run, and L. C. Ho for inspiring conversations. This work was supported by NSF grants AST-0548198 (UC Irvine), AST-0607485 and AST-0908886 (UC Berkeley), AST-0642621 (UC Santa Barbara), and AST-0507450 (UC Riverside). The UC Berkeley researchers also gratefully acknowledge the support of both the Sylvia \& Jim Katzman Foundation and the TABASGO Foundation for the continued operation of the Katzman Automatic Imaging Telescope (KAIT), with which some of the photometry was obtained. M.C.B. gratefully acknowledges support provided by NASA through Hubble Fellowship grant HF-51251 awarded by the Space Telescope Science Institute, which is operated by the Association of Universities for Research in Astronomy, Inc., for NASA, under contract NAS 5-26555. This research has made use of the Tartarus (Version 3.1) database, created by Paul O'Neill and Kirpal Nandra at Imperial College London, and Jane Turner at NASA/GSFC. Tartarus is supported by funding from PPARC, as well as from NASA grants NAG5-7385 and NAG5-7067.

\section{REFERENCES}

Alexander, D. M., et al. 2008, AJ, 135, 1968

Antonucci, R. R. J., \& Cohen, R. D. 1983, ApJ, 271, 564

Arshakian, T. G., León-Tavares, J., Lobanov, A. P., Chavushyan, V. H., Shapovalova, A. I., Burenkov, A. N., \& Zensus, J. A. 2010, MNRAS, 401, 1231

Avni, Y., \& Tananbaum, H. 1982, ApJ, 262, L17

Baldwin, J. A., Phillips, M. M., \& Terlevich, R. 1981, PASP, 93, 5

Bennert, N., Falcke, H., Schulz, H., Wilson, A. S., \& Wills, B. J. 2002, ApJ, 574, L105 
Bennert, V. N., Treu, T., Woo, J., Malkan, M. A., Le Bris, A., Auger, M. W., Gallagher, S., \& Blandford, R. D. 2010, ApJ, 708, 1507

Bentz, M. C., Peterson, B. M., Netzer, H., Pogge, R. W., \& Vestergaard, M. 2009a, ApJ, 697, 160

Bentz, M. C., Peterson, B. M., Pogge, R. W., \& Vestergaard, M. 2009b, ApJ, 694, L166

Bentz, M. C., Peterson, B. M., Pogge, R. W., Vestergaard, M., \& Onken, C. A. 2006, ApJ, 644, 133

Bentz, M. C., et al. 2007, ApJ, 662, 205

Bentz, M. C., et al. 2008, ApJ, 689, L21

Bentz, M. C., et al. 2009c, ApJ, 705, 199

Bentz, M. C., et al. 2010, ApJ, 716, 993

Blackburne, J. A., Pooley, D., Rappaport, S., \& Schechter, P. L. 2010, ApJ, submitted (arXiv:1007.1665)

Blandford, R. D., \& McKee, C. F. 1982, ApJ, 255, 419

Boroson, T. A., \& Green, R. F. 1992, ApJS, 80, 109

Collier, S. J., et al. 1998, ApJ, 500, 162

Collin, S., Kawaguchi, T., Peterson, B. M., \& Vestergaard, M. 2006, A\&A, 456, 75

Croom, S. M., et al. 2002, MNRAS, 337, 275

Dasyra, K. M., et al. 2008, ApJ, 674, L9

Davies, R. I., et al. 2006, ApJ, 646, 754

Denney, K. D., et al. 2009, ApJ, 704, L80

Denney, K. D., et al. 2010, ApJ, 721, 715

Desroches, L., Greene, J. E., \& Ho, L. C. 2009, ApJ, 698, 1515

Diamond-Stanic, A. M., Rieke, G. H., \& Rigby, J. R. 2009, ApJ, 698, 623

Dietrich, M., et al. 1994, A\&A, 284, 33

Dietrich, M., et al. 1998, ApJS, 115, 185

Ferrarese, L., Pogge, R. W., Peterson, B. M., Merritt, D., Wandel, A., \& Joseph, C. L. 2001, ApJ, 555, L79

Gallimore, J. F., et al. 2010, ApJS, 187, 172

Gaskell, C. M., \& Sparke, L. S. 1986, ApJ, 305, 175

Gebhardt, K., et al. 2000, ApJ, 543, L5

Greene, J. E., \& Ho, L. C. 2005, ApJ, 630, 122

Greene, J. E., \& Ho, L. C. 2006, ApJ, 641, L21

Greene, J. E., \& Ho, L. C. 2007, ApJ, 667, 131

Greene, J. E., \& Ho, L. C. 2009, PASP, 121, 1167

Greene, J. E., Ho, L. C., \& Ulvestad, J. S. 2006, ApJ, 636, 56

Greene, J. E., Peng, C. Y., \& Ludwig, R. R. 2010, ApJ, 709, 937

Greene, J. E., Zakamska, N. L., Liu, X., Barth, A. J., \& Ho, L. C. 2009, ApJ, 702,441

Gültekin, K., et al. 2009, ApJ, 698, 198

Hicks, E. K. S., \& Malkan, M. A. 2008, ApJS, 174, 31

Ho, L. C. 2008, ARA\&A, 46, 475

Ho, L. C., \& Peng, C. Y. 2001, ApJ, 555, 650

Hopkins, P. F., Hernquist, L., Cox, T. J., Di Matteo, T., Robertson, B., \& Springel, V. 2006, ApJS, 163, 1

Jahnke, K., et al. 2009, ApJ, 706, L215

Kaspi, S., Maoz, D., Netzer, H., Peterson, B. M., Vestergaard, M., \& Jannuzi, B. T. 2005, ApJ, 629, 61

Kaspi, S., Smith, P. S., Netzer, H., Maoz, D., Jannuzi, B. T., \& Giveon, U. 2000, ApJ, 533, 631

Kaspi, S., et al. 1996, ApJ, 470, 336

Kauffmann, G., et al. 2003, MNRAS, 346, 1055

Kellermann, K. I., Sramek, R., Schmidt, M., Shaffer, D. B., \& Green, R. 1989, AJ, 98, 1195

Kelly, B. C., Vestergaard, M., \& Fan, X. 2009, ApJ, 692, 1388

Kollatschny, W. 2003, A\&A, 407, 461

Kollmeier, J. A., et al. 2006, ApJ, 648, 128

Kuo, C. Y., et al. 2010, ApJ, submitted (arXiv:1008.2146)

Liu, X., Zakamska, N. L., Greene, J. E., Strauss, M. A., Krolik, J. H., \& Heckman, T. M. 2009, ApJ, 702, 1098

Malkan, M. A. 1983, ApJ, 264, L1

Malkan, M. A., \& Sargent, W. L. W. 1982, ApJ, 254, 22

Marconi, A., Axon, D. J., Maiolino, R., Nagao, T., Pietrini, P., Risaliti, G., Robinson, A., \& Torricelli, G. 2009, ApJ, 698, L103

Marconi, A., et al. 2008, ApJ, 678, 693

McGill, K. L., Woo, J., Treu, T., \& Malkan, M. A. 2008, ApJ, 673, 703

McHardy, I. M., Koerding, E., Knigge, C., Uttley, P., \& Fender, R. P. 2006, Nature, 444, 730

Meléndez, M., Kraemer, S. B., Schmitt, H. R., Crenshaw, D. M., Deo, R. P., Mushotzky, R. F., \& Bruhweiler, F. C. 2008, ApJ, 689, 95

Miniutti, G., Ponti, G., Greene, J. E., Ho, L. C., Fabian, A. C., \& Iwasawa, K. 2009, MNRAS, 394, 443

Morgan, C. W., Kochanek, C. S., Morgan, N. D., \& Falco, E. E. 2010, ApJ, 712 , 1129
Mulchaey, J. S., Koratkar, A., Ward, M. J., Wilson, A. S., Whittle, M., Antonucci, R. R. J., Kinney, A. L., \& Hurt, T. 1994, ApJ, 436, 586

Murray, N., \& Chiang, J. 1995, ApJ, 454, L105

Nelson, C. H., Green, R. F., Bower, G., Gebhardt, K., \& Weistrop, D. 2004, ApJ, 615,652

Netzer, H. 1990, in Active Galactic Nuclei, ed. R. D. Blandford, H. Netzer, L. Woltjer, T. J.-L. Courvoisier, \& M. Mayor (Berlin: Springer), 57

Netzer, H. 2009, ApJ, 695, 793

Netzer, H., Mainieri, V., Rosati, P., \& Trakhtenbrot, B. 2006, A\&A, 453, 525

Netzer, H., \& Marziani, P. 2010, ApJ, submitted (arXiv:1006.3553)

O’Neill, P. M., Nandra, K., Papadakis, I. E., \& Turner, T. J. 2005, MNRAS, 358, 1405

Onken, C. A., Ferrarese, L., Merritt, D., Peterson, B. M., Pogge, R. W., Vestergaard, M., \& Wandel, A. 2004, ApJ, 615, 645

Onken, C. A., et al. 2007, ApJ, 670, 105

Peng, C. Y., Impey, C. D., Ho, L. C., Barton, E. J., \& Rix, H.-W. 2006a, ApJ, 640, 114

Peng, C. Y., Impey, C. D., Rix, H.-W., Kochanek, C. S., Keeton, C. R., Falco, E. E., Lehár, J., \& McLeod, B. A. 2006b, ApJ, 649, 616

Peterson, B. M., Wagner, R. M., Crenshaw, D. M., Meyers, K. A., Byard, P. L., Foltz, C. B., \& Miller, H. R. 1983, AJ, 88, 926

Peterson, B. M., Wanders, I., Bertram, R., Hunley, J. F., Pogge, R. W., \& Wagner, R. M. 1998, ApJ, 501, 82

Peterson, B. M., et al. 1991, ApJ, 368, 119

Peterson, B. M., et al. 2000, ApJ, 542, 161

Peterson, B. M., et al. 2002, ApJ, 581, 197

Peterson, B. M., et al. 2004, ApJ, 613, 682

Salviander, S., Shields, G. A., Gebhardt, K., \& Bonning, E. W. 2007, ApJ, 662, 131

Santos-Lleó, M., et al. 2001, A\&A, 369, 57

Saxton, R. D., Read, A. M., Esquej, P., Freyberg, M. J., Altieri, B., \& Bermejo, D. 2008, A\&A, 480, 611

Schmidt, M., \& Green, R. F. 1983, ApJ, 269, 352

Schmitt, H. R., Donley, J. L., Antonucci, R. R. J., Hutchings, J. B., \& Kinney, A. L. 2003a, ApJS, 148, 327

Schmitt, H. R., Donley, J. L., Antonucci, R. R. J., Hutchings, J. B., Kinney, A. L., \& Pringle, J. E. 2003b, ApJ, 597, 768

Schulze, A., \& Wisotzki, L. 2010, A\&A, 516, A87

Searle, L., \& Sargent, W. L. W. 1968, ApJ, 153, 1003

Shapiro, K. L., et al. 2009, ApJ, 701, 955

Shen, J., Vanden Berk, D. E., Schneider, D. P., \& Hall, P. B. 2008a, AJ, 135, 928

Shen, Y., Greene, J. E., Strauss, M. A., Richards, G. T., \& Schneider, D. P. 2008b, ApJ, 680, 169

Shields, G. A. 1978, Nature, 272, 706

Shields, G. A., Gebhardt, K., Salviander, S., Wills, B. J., Xie, B., Brotherton, M. S., Yuan, J., \& Dietrich, M. 2003, ApJ, 583, 124

Silk, J., \& Rees, M. J. 1998, A\&A, 331, L1

Steffen, A. T., et al. 2006, AJ, 131, 2826

Stirpe, G. M., et al. 1994, ApJ, 425, 609

Tanaka, Y., Inoue, H., \& Holt, S. S. 1994, PASJ, 46, L37

Timmer, J., \& Koenig, M. 1995, A\&A, 300, 707

Tommasin, S., Spinoglio, L., Malkan, M. A., \& Fazio, G. 2010, ApJ, 709, 1257

Tremaine, S., et al. 2002, ApJ, 574, 740

Treu, T., Malkan, M. A., \& Blandford, R. D. 2004, ApJ, 615, L97

Treu, T., Woo, J.-H., Malkan, M. A., \& Blandford, R. D. 2007, ApJ, 667, 117

Ueda, Y., Ishisaki, Y., Takahashi, T., Makishima, K., \& Ohashi, T. 2005, ApJS, 161,185

Ulrich, M. H., et al. 1984, MNRAS, 206, 221

Uttley, P., \& McHardy, I. M. 2005, MNRAS, 363, 586

Vasudevan, R. V., \& Fabian, A. C. 2007, MNRAS, 381, 1235

Vasudevan, R. V., Mushotzky, R. F., Winter, L. M., \& Fabian, A. C. 2009, MNRAS, 399, 1553

Veilleux, S., et al. 2009, ApJS, 182, 628

Vestergaard, M., \& Osmer, P. S. 2009, ApJ, 699, 800

Vestergaard, M., \& Peterson, B. M. 2006, ApJ, 641, 689

Walsh, J. L., et al. 2009, ApJS, 185, 156

Walter, F., Carilli, C., Bertoldi, F., Menten, K., Cox, P., Lo, K. Y., Fan, X., \& Strauss, M. A. 2004, ApJ, 615, L17

Whittle, M. 1992, ApJS, 79, 49

Winge, C., Peterson, B. M., Pastoriza, M. G., \& Storchi-Bergmann, T. 1996, ApJ, 469,648

Woo, J., et al. 2010, ApJ, 716, 269

Woo, J.-H., Treu, T., Malkan, M. A., \& Blandford, R. D. 2006, ApJ, 645, 900

Woo, J.-H., Treu, T., Malkan, M. A., \& Blandford, R. D. 2008, ApJ, 681, 925

Wu, X., Wang, R., Kong, M. Z., Liu, F. K., \& Han, J. L. 2004, A\&A, 424, 793

Zhang, S., Bian, W., \& Huang, K. 2008, A\&A, 488, 113

Zheng, W., \& Malkan, M. A. 1993, ApJ, 415, 517 